\title{
Study of tau neutrino production with nuclear emulsion at CERN-SPS
}

\author{
Alina Tania Neagu ${ }^{a, *}$ on behalf of the DsTau Collaboration \\ ${ }^{a}$ Institute of Space Science, \\ Atomistilor Street, 409, Magurele Ilfov, Romania
}

E-mail: atn@spacescience.ro

The data on tau neutrino is very scarce, only a few experiments have detected its interactions. At FNAL, the beam dump experiment DONUT, the tau neutrino interaction cross-section was directly measured with a large systematical $(\sim 50 \%)$ and statistical $(\sim 30 \%)$ errors. The main source of systematical error is due to a poor knowledge of the tau neutrino flux. The effective way for the tau neutrino production is the decay of $D_{s}$ mesons, produced in proton-nucleus interactions. The DsTau experiment at CERN-SPS has been proposed to measure an inclusive differential crosssection of the $D_{s}$ production with a consecutive decay to tau lepton in p-A interactions. The goal of the experiment is to reduce the systematic uncertainty to $10 \%$ level. A precise measurement of the tau neutrino cross section would enable a search for new physics effects such as testing the Lepton Universality (LU) of Standard Model in neutrino interactions. The detector is based on nuclear emulsion providing a sub-micron spatial resolution for the detection of short length and small "kink" decays. Therefore, it is very suitable to search for peculiar decay topologies ("double kink") of $D_{s} \rightarrow \tau \rightarrow x$. After successful pilot runs and data analysis, CERN approved the DsTau project as a new experiment NA65 in 2019. During the physics runs, $2.3 \times 10^{8}$ proton interactions will be collected in the tungsten target, and about $1000 D_{s} \rightarrow \tau$ decays will be detected. In this talk, the results from the pilot run will be presented and the prospect for physics runs in 2021-2022 will be given.

\footnotetext{
*** The European Physical Society Conference on High Energy Physics (EPS-HEP2021), ***

*** 26-30 July $2021 * * *$

*** Online conference, jointly organized by Universität Hamburg and the research center DESY ***
}

\footnotetext{
${ }^{*}$ Speaker
} 


\section{Introduction}

Tau neutrino is the least studied lepton. The first detection has been announced in 2000 by DONuT experiment [1] even though the prediction of this particle was made 20 years prior [2]. $v_{\tau}$ production and detection study is important for precise measurement of its cross section. Until now only a few measurements were done in direct beam in DONuT experiment and the oscillated $v_{\tau}$ was investigated in a few experiments like OPERA [3], Super-K [4], IceCube[5]. In DONuT experiment the cross section of $D_{s}$ was estimated with a formula which depends on a few parameters: $x_{F}$ Feynman $\mathrm{x}$, the longitudinal momentum $\left(p_{Z}\right)$ and transverse momentum $\left(p_{T}\right)$.

$$
\frac{d^{2} \sigma}{d x_{F} d p_{T}^{2}} \propto\left(1-\left|x_{F}\right|\right)^{n} \exp \left(-b p_{T}^{2}\right)
$$

The formula has also a parameter $n$ that controls the longitudinal dependence of the differential production cross section, and $b$ parameter that controls the transverse dependence. Parameter $n$ was estimated only from simulations (PYTHIA) and the value used for the cross section estimation was 6.1 with an uncertainty of 50\% [6]. Taking into account the history of the tau cross section measurements, one of the physics goals of the DsTau experiment is to reduce the systematic uncertainty of $v_{\tau}$ production below $10 \%$. Other goals of the experiment are to study the tau-neutrino production in high energy proton-nuclei interactions in order to obtain the first measurement of $D_{s}$ double differential production cross section and to test Lepton Universality (LU) in neutrino interactions with $400 \mathrm{GeV}$ proton beam. This information can be a fundamental input for future $v_{\tau}$ experiments.

\section{Metodology}

All the aims mentioned above will be accomplished by detection of "double-kink + charm decay" topology within scale of a few $\mathrm{mm}$. The topology can be seen in figure 1 . We expect to detect $2.3 \times 10^{8}$ proton interactions in tungsten, and $10^{5}$ charm pairs.

Because the mean kink angle of $D_{s}$ decay is about $7 \mathrm{mrad}$, the $D_{s}$ decay to $\tau$ have a mean flight length of $3.3 \mathrm{~mm}$ and the mean flight length of tau lepton is about $2.0 \mathrm{~mm}$ [7] the detection of such short decays requires a detector with a very good spatial resolution, like nuclear emulsion. The intrinsic position resolution for nuclear emulsion is $50 \mathrm{~nm}$. The principle of detection is simple and it is based on ionization process: when particles pass through the nuclear emulsion the tracks are recorded (quasi-permanently). After the developing process (which includes amplifier and fixer steps) the trajectory of charged particle can be seen by using optical microscope.

The DsTau experimental setup consists of nuclear emulsion films, the tungsten and lead plates, and is divided into two parts: the upstream part is named the decay module and the downstream part is called Emulsion Cloud Chamber (ECC). Five additional emulsion films are placed in front of the decay module to tag the incoming beam protons. The upstream part of the target consists of a tungsten plate followed by 10 emulsion films interlaid with 200 micrometers thick plastic sheets. This unit structure is repeated 10 times to construct a module. In total 131 emulsion films are used as it can be seen in figure 2 .

The emulsion films for DsTau have two emulsion layers of $70 \mu \mathrm{m}$ thickness poured onto both sides of a $210 \mu \mathrm{m}$ thick plastic base [8]. The experimental setup contains also a silicon pixel 


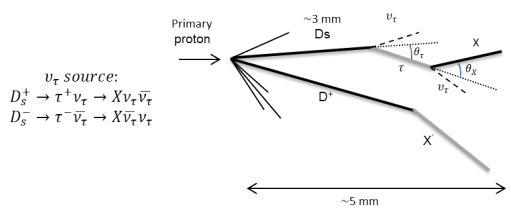

Figure 1: Topology of $D_{s}$ decay

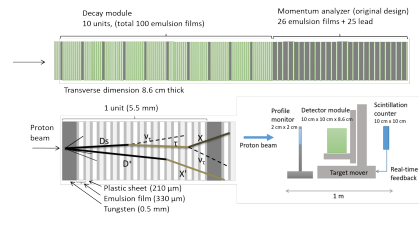

Figure 2: Experimental setup

telescope detector for beam profile monitoring, a target mover for a uniform irradiation of nuclear emulsion and the scintillation counter for a good control of intensity of the beam.

Like other detectors, nuclear emulsion benefits from the advance of technology therefore the modern emulsion detection technology is based on the remarkable evolution of the high-speed and high-precision automatic readout system, developed in Nagoya University: Hyper Track Selector (HTS) shown in figure 3. The HTS station can scan $0.5 \mathrm{~m}^{2} /$ hour with standard readout precision of track angle of about 2-5 mrad [9]. A new scanning system (HTS-2) is under development in order to increase the scanning speed to $2.5 \mathrm{~m}^{2}$ /hour.

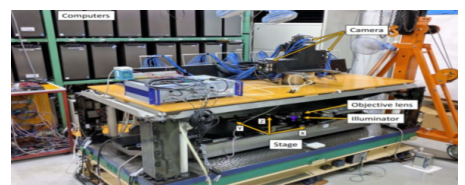

Figure 3: Hyper Track Selector (HTS)

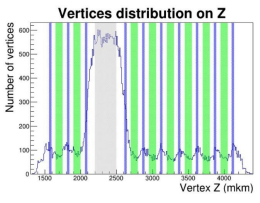

Figure 4: Reconstructed vertex position distribution in $\mathrm{Z}$

Also, the production of nuclear emulsion benefit from the advance of technology. The emulsion can be produced now on an automatic production line in Nagoya University. In this case the speed of production grows from about $6 \mathrm{~m}^{2} /$ week to $10 \mathrm{~m}^{2} /$ day.

The readout and data analysis of nuclear emulsion has few steps. The step of scanning starts with a full area scanning, followed by the detection of $\tau \rightarrow D_{s}$ decays and only then the high precision measurement is performed around the $\tau$ decay candidates to find $D_{s} \rightarrow \tau$ small kinks. After those measurements a set of selection criteria is applied for finding vertices. The selection criteria depend on the flight length of the parent, the angle between the parent and the daughter, the impact parameter of the daughter, etc. An example of reconstructed vertex position $\mathrm{Z}$ distribution can be seen in figure 4 , where a higher number of vertices in tungsten target can be identified.

\section{Status of the data analysis and prospects}

In order to investigate $D_{s} \rightarrow \tau$ decay a dedicated algorithm that searches for very small kinks using all the segments in reconstructed tracks was developed. The algorithm takes into account the small kink angles at each plate where the parent tracks of the charged decay topology passed through, calculating track angles before and after the assumed small kink point. The topology of $D_{s} \rightarrow \tau$ events appears as a double kink and another charged/neutral decay. An example of a small kink detection is shown in figure 5. An event with two large kinks was selected from data. One of the kink parents has a small kink of $2.9 \mathrm{mrad}$. 


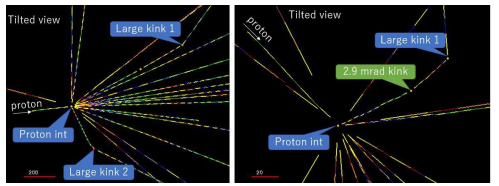

Figure 5: Example of the small kink detection

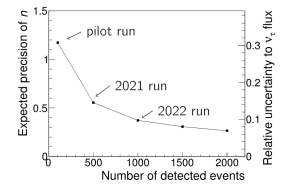

Figure 6: Prospect for DsTau experiment

For DsTau experiment a pilot run took place in 2018, another run is currently ongoing, and a third one it is planned for 2022, as can be seen in figure 6. For the pilot 2018 run the scanning of the emulsion films by the HTS is finished, the data processing is ongoing and the analyses of proton interactions and charm production are in progress.

The analysis of the data and the simulations performed for the pilot run 2018 found that it is possible to accumulate too high track density in the ECC due to the electromagnetic shower component. This fact leads to a new configuration that will be used in 2021 run with 10 units in decay part and 3 tungsten plates and 25 emulsion films for ECC.

\section{Acknowledgment}

This study was partly supported by the Romanian MCID under 16N/2019 project and Romanian - IUCN Dubna (365/11.05.2021) project no. 13 (code: 02-2-1099-2010/2023).

\section{References}

[1] K. Kodama et al., [DONuT Collaboration], Observation of Tau Neutrino Interactions, (2000) arXiv:hep-ex/0012035;

[2] M. Perl et al., Evidence for Anomalous Lepton Production in $e^{+} e^{-}$Annihilation, Phys. Rev. Lett. 35, (1975) 1489;

[3] N. Agafonova et al., Discovery of $\tau$ Neutrino Appearance in the CNGS Neutrino Beam with the OPERA Experiment, Phys. Rev. Lett. 115, (2015) 121802;

[4] Z. Li et al., [Super-Kamiokande Collaboration], Measurement of the tau neutrino cross section in atmospheric neutrino oscillations with Super-Kamiokande, Phys. Rev. D 98, (2018) 052006;

[5] M. G. Aartsen et al., [IceCube collaboration], Search for Astrophysical Tau Neutrinos in Three Years of IceCube data, Phys. Rev. D 93, (2016) 022001;

[6] K. Kodama et al., [DONuT Collaboration], Final tau-neutrino results from the DONuT experiment, Phys. Rev. D 78, (2008) 052002;

[7] S. Aoki, A. Ariga, et al., Study of tau-neutrino production at the CERN SPS, arXiv:1708.08700;

[8] S. Aoki, A. Ariga, et al., Study of tau neutrino production with $400 \mathrm{GeV}$ protons from the CERN-SPS, JHEP 2001 (2020) 033;

[9] M.Yoshimoto, T.Nakano et al., Hyper-track selector nuclear emulsion readout system aimed at scanning an area of thousand square meters, PTEP2017 no.10 (2017) $103 \mathrm{H} 01$. 